\title{
Kathon CG y Dermatología Laboral. Actualización
}

\section{Kathon CG and Occupational Dermatology. An update}

\author{
Ana Rita Rodrigues Barata ', Jesús Rodríguez Espinosa², Felipe Heras Mendaza ${ }^{3}$, Luis Conde-Salazar Gomèz*** \\ 1 Departamento de Dermatología y Venereología. Hospital Universitario Virgen Macarena. Sevilla. España. \\ 2 Fraternidad Muprespa. Cuidad Real. España \\ 3 Unidad de Dermatología Laboral. Instituto de Salud Carlos III. Pabellón 8 Universidad Complutense. Madrid. \\ España. \\ Recibido: 26-06-12 \\ Aceptado: 20-07-12

\section{Correspondencia} \\ Ana Rita Rodrigues Barata \\ Dr Fedriani, s/n. \\ 41009 Sevilla. España \\ Tfno: 605602647 \\ E-mail: Rita.rodrigues.md@gmail.com
}

\section{Resumen}

El Kathon CG constituye el nombre comercial de una mezcla de isotiazolinas: Metilcloroisotiazolinona y Metilisotiazolinona. Es un conservante muy utilizado, sobre todo en la industria cosmética, aunque en los últimos años también se ha impuesto su presencia en productos de limpieza de uso doméstico y actualmente a concentraciones más altas en preparados de uso industrial como aceites de corte, emulsiones de látex, pinturas al temple, aceites para motores Diesel, etc. Por su alto poder sensibilizante y amplia utilización, constituye actualmente una de las causas más frecuentes de alergia de contacto por preservativos, tanto en nuestra vida privada, como en el ámbito profesional.

Objetivos: Estudiar la capacidad sensibilizante del Kathon CG y su relación con el desarrollo de eczema de contacto alérgico de origen profesional.

Métodos: Estudio observacional descriptivo, a través de la revisión de las historias clínicas de los pacientes vistos en el Servicio de Dermatología Laboral del Instituto Nacional de Medicina y Seguridad en el Trabajo durante los años 2008-2012; 1520 pacientes fueron evaluados y estudiados mediante pruebas epicutáneas para descartar una posible dermatosis profesional. Se registraron los casos de sensibilización al Kathon CG y se analizaron las siguientes variables: genero, edad, grupo profesional, localización de las lesiones cutáneas, relevancia y relación profesional. El análisis estadístico se realizó con el programa SPSS 15.0.

Resultados: Se observó sensibilización al Kathon CG en 88 pacientes (5,8\%), correspondiendo un $42 \%$ a sensibilizaciones de origen profesional.

Conclusiones: La alergia de contacto profesional por Kathon CG constituye actualmente es un problema de alta prevalencia. Ante un paciente con positividad a este alérgeno hay que interrogar siempre por su profesión.

Med Segur Trab (Internet) 2012; 58 (228) 237-245

Palabras claves: Isotiazolinas, dermatitis de contacto, Kathon CG, profesional, cosméticos. 


\section{Abstract}

Kathon CG is the tradename for a mixture of isothiazolines: methylchloroisothiazolinone and methylisothiazolinone. It is a widely used preservative, especially in the cosmetic industry, although in recent years is presence have also been imposed in cleaning products for household use and, at higher concentrations in industrial preparations such as cutting oils, latex emulsions, paints, oils for diesel engines, etc. Because of its high sensitizing power and widespread use, is currently one of the most common causes of allergic contact dermatitis, both in our private lives, as in the occupational field.

Objectives: To study the sensitizing capacity of Kathon CG and its relation to the development of allergic contact dermatitis of occupational origin.

Methods: We performed a retrospective study by reviewing the medical records of patients seen at the Department of Occupationsal Dermatology at the Instituto Nacional de Medicina y Seguridad en el Trabajo, during the years 2008-2012. In that period, 1520 patients were evaluated and studied by patch testing, to rule out an occupational skin condition. We recorded cases of sensitization to Kathon CG and analyzed the following data: gender, age, occupational group, location of skin lesions, relevance and occupational origin. Statistical analysis was performed using SPSS 15.0.

Results: We observed sensitization to Kathon CG in 88 patients (5.8\%), and 42\% were of occupational origin.

Conclusions: Occupational contact allergy to Kathon CG is currently a problem of high prevalence. In patients with sensitization to this allergen, we should always ask about their occupation.

Med Segur Trab (Internet) 2012; 58 (228) 237-245

Key words: Isothiazolines, contact dermatitis, kathon CG, occupational, cosmetics. 


\section{INTRODUCCIÓN}

El Kathon CG (Cosmetic Grade) constituye el nombre comercial de una mezcla de isotiazolinas, sintetizada por la industria química a partir de los años 60 (Rohm and Haas, Filadelfia PA). Es un preservativo y potente biocida ${ }^{1}$ mu utilizado en la industria por su bajo precio y alta eficacia a bajas concentraciones frente a varios microorganismos. En su composición nos encontramos los siguientes ingredientes ${ }^{2,3}: 1,125 \%$ de Metilisotiazolinona (MI; CAS 2682-20-4) y 0,375\% de Metilcloroisotiazolinona (MCI; CAS 26172-55-4 ), que son los compuestos activos, $23 \%$ de cloruro y nitrato de magnesio, que actúan como estabilizadores y $75,5 \%$ de agua.

Su presencia ha ido en aumento desde finales de los 70 en Europa y mediados de los 80 en EE.UU, para ser, en el momento actual un preservativo ampliamente utilizado en varios ámbitos pero preferentemente en la industria cosmética, para la conservación de varios productos tales como preparados tipo rinse-off (productos que requieren aclarado, como los champús, geles de baño), y los preparados tipo leave-on (productos sin aclarado, como cremas y leches limpiadoras) ${ }^{4}$, y toallitas húmedas, a concentraciones que oscilan entre de 0,02-0,1\%. Además es muy relevante la presencia de este preservativo en productos de limpieza de uso doméstico y a concentraciones más elevadas en productos de utilización industrial, como los aceites de corte, emulsiones de látex, pinturas al temple, reveladores de fotografía y radiografía, aceites para motores diesel, pesticidas, abrillantadores, tintas de imprenta... ${ }^{5-9}$.

Asimismo es conocido por ser un potente sensibilizante y los primeros casos de dermatitis de contacto por Kathon CG a partir de cosméticos se publicaron ya en $1985^{10,11}$, y desde entonces inúmeros casos fueron reportados en la literatura. Por su amplia y generalizada utilización, tanto en el ámbito privado como en la vida profesional, el Kathon CG es actualmente una de las causas más frecuentes de alergia de contacto por preservativos.

La misma mezcla de isotiazolinas está comercializada por otros fabricantes bajo diferentes nombres comerciales, como por ejemplo: Acticide, Algucid CH50, Amerstat 250, Euxyl K 100, Fennosan IT 21, Grotan K, Grotan TK2, Mergal K7, Metat GT, Metatin GT, Paretol, Parmetol... y el propio fabricante de Kathon CG comercializa otras mezclas de MCI/MI a concentraciones más elevadas ${ }^{12}$.

\section{MATERIAL Y MÉTODOS}

Con el objetivo de estudiar la sensibilización al Kathon CG en el medio laboral, se realizó un estudio observacional descriptivo y retrospectivo durante el periodo de enero de 2008 y mayo de 2012. Se utilizaron como fuentes de datos las historias clínicas de los pacientes vistos en el Servicio de Dermatología Laboral del Instituto Nacional de Medicina y Seguridad en el Trabajo (INMST), que se encuentran almacenados en un programa informático Lotus 97.

Se estudiaron las siguientes variables: epidemiológicas (género y edad), profesión, localización del proceso cutáneo, alérgenos positivos, relevancia clínica y relación profesional. A todos los pacientes se les realizó una anamnesis detallada, haciendo hincapié en todos los preparados y productos utilizados por el paciente en el ámbito de su vida privada y los manejados durante el periodo laboral.

Durante el periodo de estudio acudieron a nuestro centro un total de 1520 pacientes, derivados para estudio de una posible dermatosis profesional. A todos los pacientes se les realizó pruebas epicutáneas de la bacteria estándar del GEIDAC (Grupo Español de Investigación de Dermatitis Alérgica de Contacto), baterías específicas en función del cuadro clínico y profesión del paciente, y productos propios. Los alérgenos utilizados provenían de la marca comercial TRUE TEST®, suministrado por Marti-Tor y de CHEMOTECHNIQUE DIAGNOSTICS y colocados en los parches curatest ${ }^{\circ}$. El Kathon CG 
se encuentra a una concentración de 100 ppm en agua. Las pruebas epicutáneas se colocaron en espalda superior, fijados mediante tiras adhesivas. Se levantaron a las 48 horas para una primera lectura y a las 96 horas para una segunda y definitiva lectura, siguiendo la normativa del ICDRG (International Contact Dermatitis Research Group).

\section{RESULTADOS}

Se parchearon un total de 1520 pacientes durante el periodo en estudio, y el número de sensibilizaciones al Kathon CG fue de 88 pacientes (5,8\%). Del total, 47 eran mujeres y 41 hombres ( $53 \%$ y $47 \%$ respectivamente) (Figura 1), observándose todos los años, con excepción de 2010, un mayor número de mujeres afectadas. Respecto a edad de los pacientes, se observó un rango entre 19 a 70 años, con una media de 42,8 años de edad, y el $55 \%$ presentaban edades comprendidas entre 35 y 55 años, lo que corresponde a la mitad de la vida laboral de la población activa (Figura 2). Además se observó que la aparición de los síntomas cutáneos fue más precoz en el genero femenino, con una media de 41,3 años frente a 44,5 años en el genero masculino.

Figura 1. Distribución por género de las positividades al Kathon CG

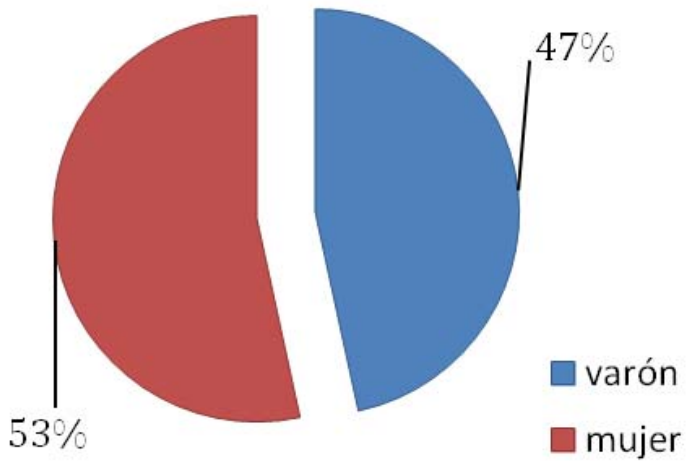

Figura 2. Distribución de casos por edad y género

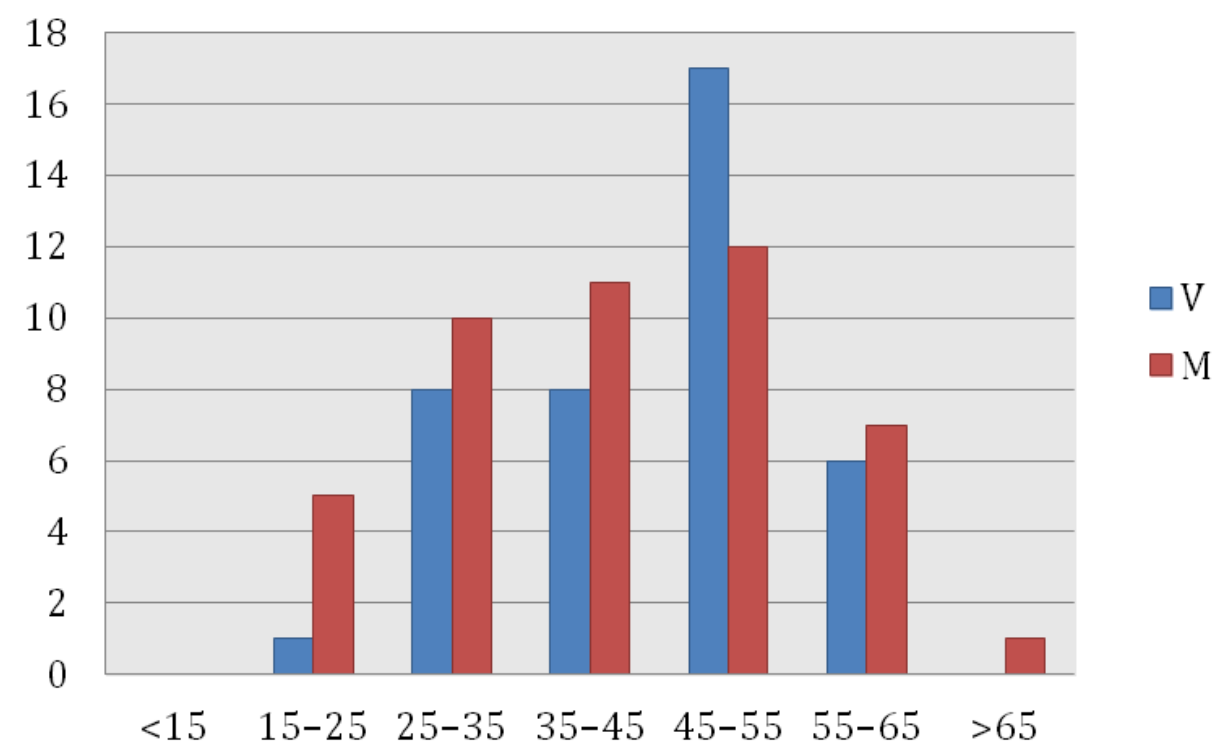


Se ha observado un $67 \%$ de polisensibilizaciones, siendo el sulfato de níquel el $2^{\mathrm{a}}$ alérgeno más frecuente con un 50,8\%. De los 29 pacientes que dieron solamente positividad frente a Kathon y no a otros agentes sensibilizantes, 16 eran hombres (55.2\%) y el resto mujeres ( $44.8 \%)$.

La localización del proceso cutáneo fue más frecuente en las manos con un 75\%, con distribución proporcional en dorso, palmas y superficies digitales, aunque observamos presencia de lesiones cutáneas a nivel de todo el tegumento cutáneo, incluso 2 casos de dermatitis de contacto aerotransportada (Tabla 1).

Tabla 1. Distribución de Localizació de lesiones cutáneas

\begin{tabular}{lcc}
\hline \multicolumn{1}{r}{ Localización } & N: 88 & $\%$ \\
\hline Manos & 66 & 75 \\
Dorso & 24 & \\
Palmas & 22 & \\
Dedos & 20 & \\
Cabeza y cuello & 8 & 9,1 \\
Generalizado & 6 & 6,8 \\
Extremidades & 6 & 6,8 \\
Tronco & 2 & 2,3 \\
\hline
\end{tabular}

En relación a la profesión, hemos obtenido un amplio rango de grupos profesionales aunque destacan 3 grupos mas prevalentes, que por orden decreciente fueron: industria automotriz/metalurgia (19,1\%), sanitarios $(17,3 \%)$ y peluquería $(12,5 \%)$ (Tabla 2). Destacar que dentro del grupo sanitario, el 53,3 \% correspondían con Auxiliares de Geriatría.

Tabla 2. Relación de casos positivos por Grupos Profesionales

\begin{tabular}{lcc}
\hline \multicolumn{1}{c}{ Profesión } & N: 88 & $\%$ \\
\hline Mecánica/Metalúrgia & 17 & 19,3 \\
Sanitarios & 15 & 17,1 \\
Peluqueria & 11 & 12,5 \\
Construcción & 8 & 9,1 \\
Artes gráficas & 8 & 9,1 \\
Administrativos & 7 & 7,9 \\
Limpieza & 6 & 6,8 \\
Miscelania & 16 & 18,2 \\
\hline (Tto de aguas, Cocina, Comercial, Almacén, Hostelería, \\
Mantenimiento, Pintor/Chapista, Químico, Floristería, \\
Músico, Profesora de danza, Masajista, Panadero). \\
\hline
\end{tabular}

Respecto de la relevancia de las pruebas epicutáneas, observamos una relevancia presente en 78 pacientes (88\%), y de estas 33 (43\%) fueron de origen profesional. Podemos sumarles otros 13 casos (17\%) en los que se encontró sustancias sensibilizantes tanto en el medio laboral como en la vida privada, por lo que no se pudo asegurar que la sensibilización fuera solamente de origen profesional. (Figura 3). 
Figura 3. Distribución de Relevancia de las pruebas epicutáneas

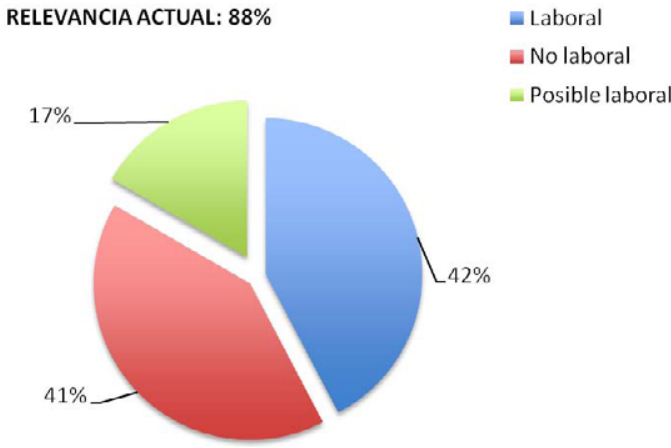

\section{DISCUSIÓN}

Los preservativos son aditivos indispensables en la manufacturación de productos con base acuosa, ya que evitan el sobrecrecimiento de microorganismos ${ }^{2,13}$. Por esta razón se utilizan en el proceso de fabricación de cosméticos, productos de limpieza y determinados productos industriales. La MCI y la MI, al igual que otros conservantes del grupo formaldehído o mercuriales, pueden tener alto poder de sensibilización, como se ha demostrado por estudios experimentales y por la practica clínica, presentando la MCI mayor poder sensibilizante que la $\mathrm{MI}^{12}$. Actualmente constituye una de las causas más frecuente de alergia de contacto por preservativos ${ }^{14-16}$.

Asimismo, además de la MCI y MI existen otras isotiazolinas disponibles para la producción de productos industriales, en particular, la benzisotiazolinona, que se utiliza con frecuencia en la pintura, pudiendo ser responsable de reacciones cruzadas con $\mathrm{MCI} / \mathrm{MI}^{17}$.

La concentración de $\mathrm{MCI} / \mathrm{MI}$ en productos cosméticos ha estado regulada desde los años 80, sin embargo sin presentar impacto en la prevalencia de su sensibilización en la población general ${ }^{16,18}$. Bajo la legislación actual la concentración máxima de Kathon CG es de 15 ppm en todos los cosméticos en la Comunidad Europea, mientras que en los EEUU se establece como concentración máxima para los preparados rinse-off $15 \mathrm{ppm}$ y para los leave-on 7,5 $\mathrm{ppm}^{19,20}$, siendo estas las recomendaciones del fabricante ${ }^{21}$.

Aceptando de entrada que se cumple la normativa establecida, las elevadas cifras de prevalencia de sensibilización a este alérgeno se podrán explicar por varias hipótesis: en parte por el alto consumo actual de productos cosméticos, sobre todo en pacientes con dermatitis preexistentes, que al presentar una barrera cutánea alterada son más susceptibles a sensibilizarse frente a varios alérgenos, como queda de manifiesto en la revisión efectuada por Conti et $a^{22}$ en consultas de Dermatología pediátrica, donde el Kathon es el alérgeno más frecuente en este grupo de edad.

Por otra parte otros factores podrían influir teóricamente en la aparición de esta sensibilización: se produce una sumación de la concentración del Kathon al aplicar más de un producto sobre la misma superficie cutánea; y además la aplicación puede ser en piel, mucosas o semimucosa tanto sana como con alteraciones de la barrera cutánea, y bajo oclusión, lo que aumentaría la penetración del alérgeno y posibilidad de sensibilización ${ }^{23,24}$

A pesar del gran numero de nombres comerciales de preparados que contienen MCI/MI (en 1992 había registrados 550 en el mercado internacional), y la imposibilidad de conocerlos a todos, debemos insistir a nuestros pacientes, que lo que deben recordar 
es el nombre de "Isotiazolina" que es como debe aparecer en el etiquetado de los varios preparados comerciales desde enero de 1998 en todos los cosméticos, sean de perfumería o farmacia ${ }^{22,24}$.

El eczema de contacto es el cuadro clínico más frecuente observado en pacientes con sensibilización a isotiazolinas, pero se pueden observar otras presentaciones clínicas, como urticarias de contacto, dermatitis aerotransportada ${ }^{25}$, quemaduras con sensibilización posterior $^{26}$ y reacciones generalizadas tras exposición a productos que lo contengan, incluso tras periodos de tiempo largo, como Fernández De Corres et al ${ }^{27}$ que describieron un paciente con una erupción generalizada tras entrar en su puesto de trabajo pintado hacía varios meses con pintura al temple que contenía Kathon. Además actualmente está en estudio la capacidad de teratógena y oncogénica de estas sustancias ${ }^{28,29 .}$

Un estudio multicéntrico de prevalencia efectuado a nivel Europeo mostró valores de sensibilización muy dispares, con cifras que oscilan entre $0,4 \%$ en el Reino Unido y 11,1\% en Italia ${ }^{30}$. En 1990 en este mismo Servicio de Dermatología Laboral se realizó el primer estudio de sensibilización al Kathon CG en nuestro país, encontrando los primeros 22 casos que suponían un 3,5\% de las positividades ${ }^{31}$. Posteriormente se realizaron estudios similares, para seguir la tendencia de la prevalencia de la sensibilización de este preservativo, y se obtuvieron cifras de $2,89 \%$ en un estudio realizado entre $1990-1997^{32}$, y $4,71 \%$ durante los años de 2003-2006 33 .

En relación a la sensibilización al Kathon CG en el medio profesional, se sabe que este preservativo está presente en numerosas sustancias utilizadas en este ámbito. Actualmente no hay restricciones en su uso en productos industriales y se utilizan a mayores concentraciones de las que se permiten para productos cosméticos. Asimismo existen pocas publicaciones sobre sensibilización profesional a este preservativo. En el presente estudio, observamos una sensibilización al Kathon CG de origen profesional presente en el $42 \%$ de los casos. Además de este valor queda un $17 \%$ de sensibilizaciones en las cuales no esta claro si realmente la sensibilización es profesional, pero podríamos afirmar que el trabajo influye bien como sensibilizante inicial o como promotor del cuadro dermatológico. En la ultima revisión sobre sensibilización al Kathon en nuestro centro se observaron valores de origen profesional similares ${ }^{33}$. Estas cifras nos permiten afirmar que un porcentaje importante de la sensibilización al Kathon es de origen profesional.

\section{CONCLUSIONES}

Según nuestro estudio, la sensibilización al Kathon en los cuatros últimos años en nuestro Centro fue de 5,8\%, cifra más elevada que las obtenidas en estudios anteriores, con un origen profesional en el $42 \%$ de los casos. Además se observó un $17 \%$ de sensibilizaciones en el que no se pudo confirmar el origen laboral de la sensibilización, pero en estos casos si se puede afirmar que bien influye como sensibilizante inicial o como promotor del cuadro dermatológico.

La sensibilización al Kathon CG debe ser considerada una dermatosis profesional importante, por lo que ante todo el paciente con positividad a este alérgeno hay que interrogar por su profesión. Asimismo es necesario establecer una normativa en el ámbito industrial que regule la concentración máxima de Kathon CG o sustituirlo por otros preservativos con menor capacidad de sensibilización de forma a disminuir la prevalencia de este problema. 


\section{REFERENCIAS BIBLIOGRÁFICAS}

1. Hunziker N. The isothiazoline story. Dermatology. 1992;184:85-86.

2. Marks J G, Moss J N, Parno J R, Fowler J F, Storrs, F J, Belsito D V, et al.: Methylchloroisothiazolinone/ Methylisothiazolinone (Kathon CG) Biocide- United. States Multicenter Study of Human Skin Sensitization. Am J Contact Dermatitis 1990; 3: 157-161.

3. Marks J G, Moss J N, Parno J F, Adams R M, Belsito D V, De Leo F, et al.: Methylchlorothiazolinone/ Methylisothiazolinone (Kathon CG) Biocide: Second. United States Multicenter Study of Human Skin Sensitization. Am J Contact Dermatitis 1990; 4: 87-89.

4. Rastogi SC. Kathon CG and cosmetic products. Contac Dermatitis. 1990 Mar;22:155-60.

5. Fischer T, Bohlin S, Edling C, Rystedt I, Wieslander G. Skin disease and contact sensitivity in house painters using water-based, glues and putties. Contact Dermatitis 1995; 32: 39-45.

6. Rycroft R J G, Neil V S. Allergic contact dermatitis from MCI/MI biocide in a printer. Contact Dermatitis 1992; 26: 142-143.

7. Bruynzeel D P, Verburgh C A. Occupational dermatitis from isothiazolinones in diesel oil. Contact Dermatitis 1996; 34: 64-65.

8. Pazzaglia M, Vincenzi C, Gasparri F, Tosti A. Occupational hypersensitivity to isothiazolinone derivatives in a radiology technician. Contact Dermatitis 1996; 34: 143.

9. Guimaraens D, Conde-Salazar L, González M A. Allergic contact dermatitis on the hands from chloromethylisothiazolinone in moist toilet paper. Contact Dermatitis 1996; 35: 254.

10. De Groot AC, Liem DH, Nater JP et al. Patch tests with fragrance materials and preservatives. Contact Dermatitis 1985; 12:87-92.

11. De Groot AC, Liem DH, Weyland JW. Kathon CG: cosmetic allergy and patch test sensitization. Contact Dermatitis 1985; 12:76-80.

12. De Groot A C. Methylisothiazolinone/Methylchloroisotiazolinone (Kathon CG) Allergy: An Updated review. Am J Contact Dermatitis 1990; 3: 151- 156.

13. Nielsen H. Occupational exposure to isothiazolinones. A study based on a product register. Contact Dermatitis 1994; 31: 18-21.

14. Schnuch A, Lessmann H, Geier J et al. Contact allergy to preservatives. Analysis of IVDK data 1996-2009. Br J Dermatol 2011; 164:1316-25.

15. Wilkinson JD, Shaw S, Andersen KE et al. Monitoring levels of preservative sensitivity in Europe. A 10-year overview (1991-2000). Contact Dermatitis 2002; 46:207-10.

16. Thyssen JP, Engkilde K, Lundov MD et al. Temporal trends of preservative allergy in Denmark (19852008). Contact Dermatitis 2010; 62:102-8.

17. Isaksson M, Bruze M, Gruvberger B. Cross-reactivity between methylchloroisothiazolinone / methylisothiazolinone, methylisothiazolinone, and other isothiazolinones in workers at a plant producing binders for paints and glues. Contact Dermatitis 2008; 58:60-2.

18. Marks JG Jr, Belsito DV, DeLeo VA et al. North American Contact Dermatitis Group patch-test results, 1996-1998. Arch Dermatol 2000; 136:272-3.

19. European Commission. Council Directive 76/768/EEC of 27 July 1976 on the approximation of the laws of the Member States relating to cosmetic products (consolidated version 24-4-2008). (last accessed 9 May 2012) Available at: http://ec.europa.eu/enterprise/cosmetics/html/consolidated_dir.htm.

20. U.S. Food and Drug Administration. Federal Food, Drug, and Cosmetic Act. 31-12-2004. (last accessed 12 April 2012) Available at: http://www.fda.gov/RegulatoryInformation/Legislation/FederalFoodDrugand CosmeticActFDCAct/ default.htm.

21. Rohm and Haas. Kathon CG: A safe, effective, globally approved preservative for rinse-off products. 2006. (last accessed 12 April 2012). Available at: http://www.rohmhaas.com/assets/attachments/business/ pcare/kathon_for_personal_care/kathon_cg/tds/kathon_cg.pdf.

22. Conti A, Motolese A, Manzini B M, Seidenari S. Contact sensitization to preservatives in children. Contact Dermatitis 1997; 37: 35-52.

23. De Groot A C. Vesicular dermatitis of the hands secondary to perianal allergic contact dermatitis caused by preservatives in moistened toilet tissues. Contact Dermatitis 1997; 36: 173.

24. Gruvberger B, Persson K, Bjorkner B, Bruze M, Dahlquist I, Fregert S. Demonstration of Kathon CG in some comercial products. Contac Dermatitis. 1986 Jul;15:24-7. 
25. Schubert H. Airborne contact dermatitis due to methylchloro-and methylisothiazolinone (MCI/MI). Contact Dermatitis 1997; 36: 274.

26. Monsálvez V, Fuertes L, Ortiz de Frutos J, Vanaclocha F. Sensitization to MethylchoroisothiazolinoneMethylisothiazolinone After a Burn Caused by Massive Accidental Occupational Exposure. Actas Dermosifiliogr. 2011 Apr;102:224-226.

27. Fernández De Corres L, Navarro J A, Gastaminza G, Del Pozo M D. An unusual case of sensitization to methylchloro- and methylisothiazolinone (MCI/MI). Contact Dermatitis 1995; 33: 215.

28. Longacre S L. Genetic toxicity and carcinogenicity studies of Kathon. Environ Mol Mutagen 1997; 29, 3: 331-333.

29. Comnor TH, Tee PG, Afshar M, Comnor KM. Mutagenicity of cosmetic products containing Kathon Mutagen 1997;29(3):330.

30. Menné T, Frosch P J, Veien N K, Hannuksela M, Björkner B, Lachapelle J M, et al. Contact sensitization to 5-chloro-2methyl-4-isothiazolin-3-oneand 2-methyl-4- isothiazolin-3 one and 2-methyl-4-isothiazolin3-one (MCI/MI). A European multicenter study. Contact Dermatitis 1991; 24: 333-341.

31. Hasson A, Guimaraens D, Conde-Salazar L. Patch test sensitivity to the preservative Kathon CG in Spain. Contact Dermatitis 1990; 22: 257-261.

32. Conde-Salazar L, Flis M, González MA, Guimaraens D. Sensitization to Kathon CG in Occupational Dermatology Service (Review 1990-1997). Rev Esp Alergol Inmunol Clin 1998;13:268-272.

33. Cabrera Fernández E, Moreno Manzano G, Barchino Ortiz L, Perez García V, Heras Mendaza F, CondeSalazar L. Isotiazolinas: Importancia en el desarrollo de dermatitis de contacto professional. Med Segur Trab 2007;Vol LIII n ${ }^{\circ}$ 208:00-00.

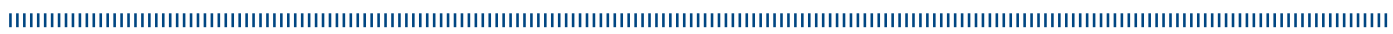

